\title{
Evolución de la Desigualdad en la Mortalidad Infantil
}

\author{
MARIANA FLORES F. ${ }^{1}$, JAIME CERDA L. ${ }^{2}$
}

1. Departamento de Pediatría, Facultad de Medicina, Pontificia Universidad Católica de Chile.

2. Departamento de Salud Pública, Facultad de Medicina, Pontificia Universidad Católica de Chile.

\begin{abstract}
Evolution of infant mortality rate disparity among districts of the Metropolitan Region, Chile

Background: In Chile, infant mortality rate (IMR) reduced substantially during the last decades; however, the trend of the disparity between districts with different socioeconomic status (SES) is unknown. Objective: Describe the trend of the disparity of IMR. Methods: Ecologic study. For measuring IMR disparity the following indicators were calculated: population attributable risk (PAR), population attributable risk percent (PAR\%), rate ratio (RR) and districtual attributable risk (AR). The first three permitted a global comparison (Chile $v s$ high SES district), while the fourth permitted specific comparisons (9 districts of the Metropolitan Region $v s$ high SES district). CASEN poverty index was used for characterizing SES. Results: Globally, PAR decreased from 8.3 (1990) to 3.5 per 1,000 (2006), while RR decreased from 1.98 (1990) to 1.80 (2006). Specifically, both districtual AR and poverty indexes in the 9 studied districts became more homogeneous in time, reflecting a decrease in the disparity gap. Conclusion: Between 1990 and 2006 IMR disparity among districts of the Metropolitan Region decreased, coincidently with a reduction in poverty levels. Nevertheless, important gaps still persist.
\end{abstract}

(Key words: Infant mortality, disparity, poverty, Chile).

Rev Chil Pediatr 2010; 81 (3): 215-220

\section{RESUMEN}

Antecedentes: En Chile, la tasa de mortalidad infantil (TMI) se redujo sustancialmente durante las últimas décadas, sin embargo, se ignora la evolución temporal de la desigualdad entre comunas de diferente nivel socioeconómico (NSE). Objetivo: Describir la evolución temporal de la desigualdad en la TMI. Métodos: Estudio ecológico. Para medir la desigualdad en la TMI se calculó: riesgo atribuible poblacional (RAP), riesgo atribuible poblacional porcentual (RAP\%), razón de tasas (RT) y riesgo atribuible comunal (RA). Los tres primeros indicadores permitieron realizar comparaciones globales (Chile $v s$ comuna de NSE alto) y el cuarto

Trabajo recibido el 08 de enero de 2010, devuelto para corregir el 03 de marzo de 2010, segunda versión el 24 de mayo de 2010, aceptado para publicación el 26 de mayo de 2010.

Correspondencia a:

Dr. Jaime Cerda L.

E-mail: jcerda@med.puc.cl 
comparaciones específicas (comunas de la Región Metropolitana vs comuna de NSE alto). El NSE se caracterizó mediante el índice de pobreza CASEN. Resultados: Globalmente, el RAP disminuyó de 8,3 (1990) a 3,5 por $1000 \mathrm{NV}$ (2006); por su parte, la RT se redujo de 1,98 (1990) a 1,80 (2006). Específicamente, tanto los RA comunales como los índice de pobreza de las 9 comunas estudiadas se tornaron más homogéneos en el tiempo, reflejo de una reducción de la desigualdad. Conclusión: Entre 1990 y 2006 la desigualdad en la TMI entre comunas de la Región Metropolitana disminuyó, coincidentemente con una reducción de la pobreza. Sin embargo, aún persisten importantes brechas.

(Palabras clave: Mortalidad infantil, Desigualdad, Pobreza, Chile).

Rev Chil Pediatr 2010; 81 (3): 215-220

\section{Introducción}

El término equidad hace referencia a la ausencia de desigualdades injustas y evitables en el estado de salud de las personas, tanto a nivel individual como colectivo. Las desigualdades en salud no se producen al azar, por el contrario, el estado de salud guarda relación con las características biológicas y sociales de los individuos, la organización económica y política, la estructura social y el trasfondo cultural, como también con los procesos demográficos y macroecológicos ${ }^{1,2}$. Medir las desigualdades es el primer paso para identificar las inequidades en el ámbito de la salud, analizar sus determinantes y plantear una teoría, base fundamental para un plan de acción.

La tasa de mortalidad infantil (TMI) es un buen indicador para resumir la infraestructura sanitaria de una población y las condiciones ambientales, socioculturales y económicas que pueden actuar negativamente sobre la salud de las personas. En Chile, esta tasa se ha reducido en forma sustancial durante las últimas décadas, desde 86,5 por $1000 \mathrm{NV}$ (1970) a 7,9 por $1000 \mathrm{NV}(2006)^{3}$, situando al país en un lugar destacado en el contexto latinoamericano. Sin embargo, por su carácter global la TMI oculta desigualdades existentes entre comunas de diferente nivel socioeconómico, las cuales adquieren el carácter de inequidad toda vez que son injustas y evitables. Tal realidad ha sido objetivada por Donoso, quien en base a datos de 2000 demostró una asociación directa entre el nivel de pobreza comunal y su respectiva $\mathrm{TMI}^{4}$. Esta realidad no ha sido ignorada por la autoridad sanitaria; al respecto, dos Objetivos Sanitarios para la Década 2000-2010 tienen directa relación con mortalidad infantil, siendo el primero reducir en $25 \%$ la TMI, desde 10,1 por $1000 \mathrm{NV}$ (1999) a 7,5 por $1000 \mathrm{NV}$ (2010) y el segundo reducir la brecha de TMI entre quintiles extremos de escolaridad materna, la cual ha mantenido estable su riesgo relativo de 5,0 desde $1970^{5}$.

El objetivo del presente estudio fue caracterizar la evolución de la desigualdad en la TMI de diferentes comunas de la Región Metropolitana, utilizando medidas y metodologías económicas y epidemiológicas sencillas, aplicadas a un conjunto de indicadores recogidos por sistemas rutinarios de información. El fin último de este estudio fue proporcionar información útil sobre las brechas existentes, facilitando la génesis e implementación de intervenciones eficaces que garanticen la equidad en la provisión y acceso a los servicios de salud, focalizada en los sectores más necesitados.

\section{Material y Métodos}

\section{Evaluación de la desigualdad de la tasa de mortalidad infantil}

\section{Comparación global}

Para medir la desigualdad global de la TMI a nivel nacional se utilizaron tres indicadores, a saber, riesgo atribuible poblacional (RAP), riesgo atribuible poblacional porcentual (RAP\%) y razón de tasas $(\mathrm{RT})^{6}$. El RAP se calculó según la fórmula RAP $=$ (TMI Chile-TMI comuna ' $\mathrm{X}$ '), correspondiendo esta última a la comuna con el menor índice de pobreza en 2006 dentro de aquellas incluidas en el estudio. Conceptualmente, el RAP es equivalente al número de muertes de menores de 1 año por $1000 \mathrm{NV}$ 
potencialmente evitables en el escenario hipotético que todas las comunas de Chile tuviesen las mismas características que la comuna ' $\mathrm{X}$ '. Por su parte, el RAP\% se calculó según la fórmula RAP $\%=(\mathrm{RAP} / \mathrm{TMI} \text { Chile })^{*} 100$, siendo equivalente al porcentaje de la TMI de Chile que podría reducirse en el escenario hipotético que todas las comunas del país tuviesen las mismas características que la comuna ' $\mathrm{X}$ '. Por último, la RT se calculó según la fórmula RT $=(\mathrm{TMI}$ Chile/TM comuna ' $\mathrm{X}$ '), siendo equivalente al número de veces en que la TMI de Chile supera la TMI de la comuna ' $X$ '. Los tres indicadores están directamente asociados al nivel de desigualdad existente.

\section{Comparación específica (intercomunal)}

Para medir la desigualdad específica de la TMI se calculó el riesgo atribuible comunal (RA), según la fórmula $\mathrm{RA}=(\mathrm{TMI}$ comuna ' $Y_{n}$ '-TMI comuna ' $X$ '), correspondiendo ' $Y_{n}$ ' a la comuna en estudio y ' $\mathrm{X}$ ' a la comuna con el menor índice de pobreza en 2006 entre las incluidas en el estudio. Conceptualmente, el
RA es equivalente al número de muertes de menores de 1 año por $1000 \mathrm{NV}$ potencialmente evitables en el escenario hipotético que la comuna ' $\mathrm{Y}_{\mathrm{n}}$ ' tuviese las mismas características que la comuna ' $\mathrm{X}$ '.

\section{Fuentes de información}

La tasa de mortalidad infantil (i.e. muertes en menores de 1 año/1 000 nacidos vivos inscritos) de Chile y de las comunas incluidas fueron obtenidas a partir de los Anuarios de Demografía, publicados por el Instituto Nacional de Estadísticas 7 . Por su parte, el índice de pobreza (i.e. porcentaje de personas que viven en condiciones de pobreza, indigentes y pobres no-indigentes) se obtuvo a partir de los datos reportados por la Encuesta de Caracterización Socioeconómica (CASEN), realizada por el Ministerio de Planificación y Cooperación ${ }^{8}$. Se registraron datos incluidos en el período 19902006, cada 4 años (1990, 1994, 1998, 2003 y 2006). Se incluyó el año 2003 en lugar del 2002 debido a que la encuesta CASEN se realizó en el primero, no así en el segundo.

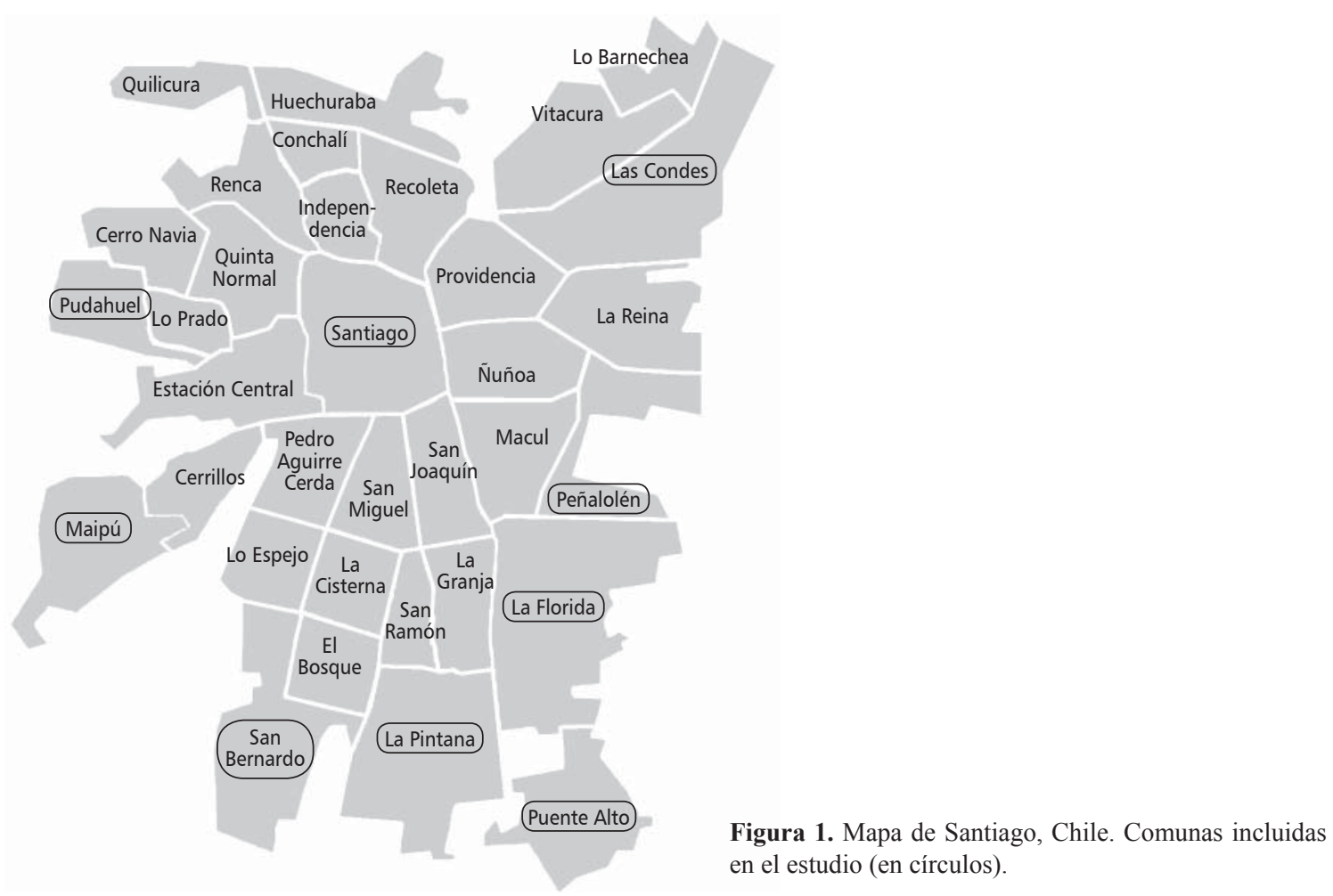



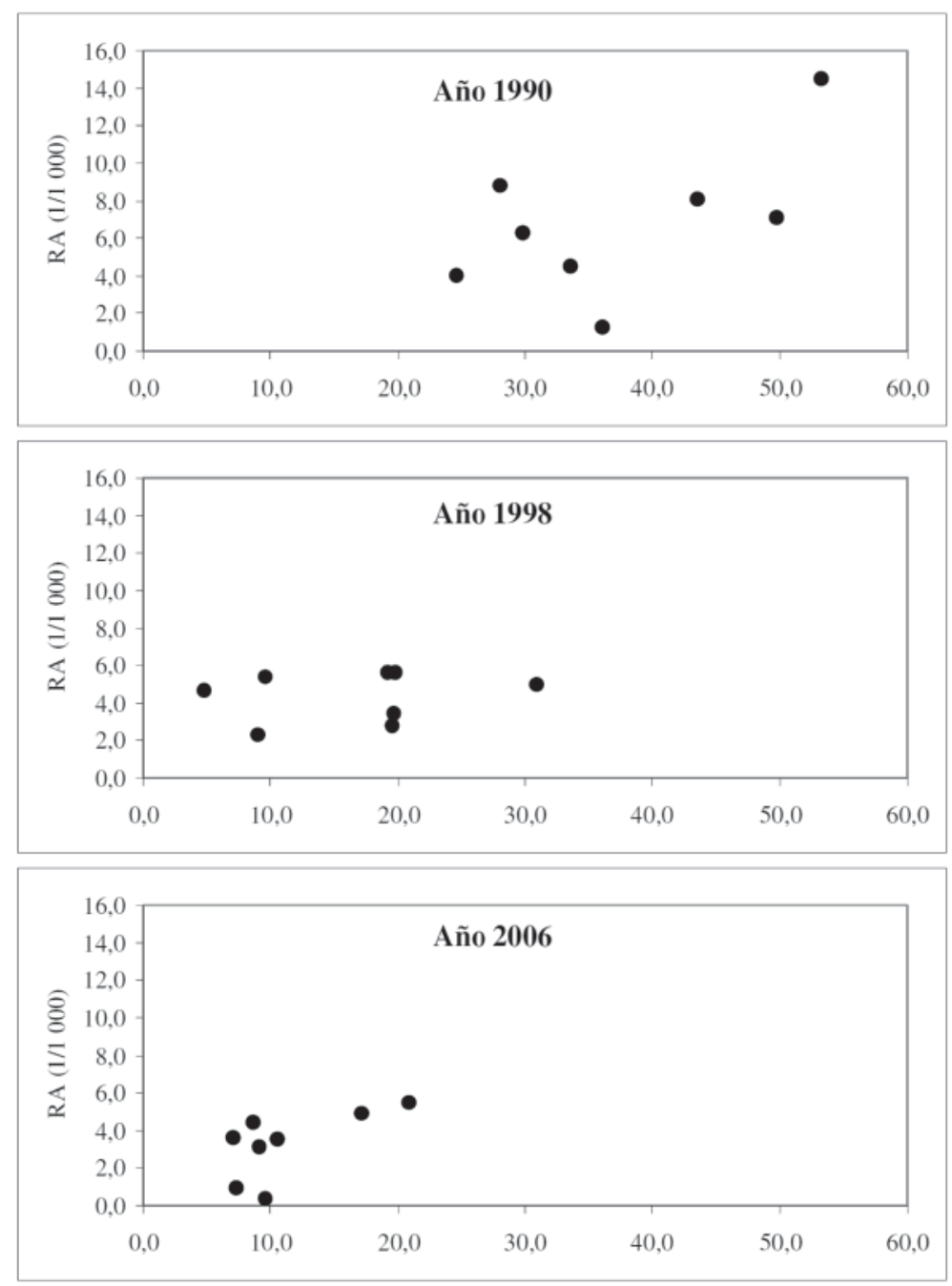

Indice de Pobreza (1/100)
Figura 2. Índice de Pobreza (CASEN) vs riesgo atribuible de Mortalidad Infantil de 9 comunas de la Región Metropolitana, según año. ${ }^{*}$ Comunas: La Florida, La Pintana, Maipú, Peñalolén, Pudahuel. Puente Alto, San Bernardo y Santiago. Comuna de Referencia: Las Condes.

\section{Selección de las comunas}

Fueron incluidas las comunas de la Región Metropolitana que 2006 tuviesen (a) una población mayor a 175 mil habitantes y (b) datos completos referentes a tasa de mortalidad infantil. Las comunas seleccionadas fueron (en orden alfabético): La Florida (395 mil h), La Pintana (201 mil h), Las Condes (274 mil h), Maipú (663 mil h), Peñalolén (238 mil h), Pudahuel (237 mil h). Puente Alto (627 mil h), San Bernardo (286 mil h) y Santiago (182 mil h) (figura 1).

\section{Resultados}

\section{Comparación global (Chile vs comuna de} NSE alto)

Para el año 2006, la comuna con el menor índice de pobreza (CASEN) fue la comuna de Las Condes $(2,3 \%)$, siendo considerada como el estándar de referencia. En 1990 la TMI de Chile fue 16,8 por $1000 \mathrm{NV}$, mientras que en la comuna de Las Condes fue 8,5 por 1000 $\mathrm{NV}$; por consiguiente, el RAP fue 8,3 por 1000 NV y la RT 1,98. En términos absolutos, 2428 
muertes de menores de 1 año son atribuibles al hecho de que las comunas que integran Chile no presentaban las características de la comuna de Las Condes. Estas defunciones equivalen al $49,4 \%$ de las muertes de menores de 1 año registradas en Chile en 1990 (RAP\%). Dieciséis años más tarde (2006), la TMI de Chile fue 7,9 por $1000 \mathrm{NV}$, mientras que en la comuna de Las Condes fue 4,4 por $1000 \mathrm{NV}$; por consiguiente, el RAP fue 3,5 por $1000 \mathrm{NV}$ y la RT 1,80. En términos absolutos, 815 muertes de menores de 1 año son atribuibles al hecho de que las comunas que integran Chile no presentaban las características de la comuna de Las Condes. Estas defunciones equivalen al 44,3\% de las muertes de menores de 1 año registradas en Chile en 2006 (RAP\%).

\section{Comparación específica (Intercomunal)}

La figura 2 ilustra los valores de índice de pobreza (CASEN) versus el RA de las 9 comunas incluidas en el estudio, calculados en los años 1990, 1998 y 2006, considerando a la comuna de Las Condes como el estándar de referencia. La secuencia de figuras comienza en el año 1990, en el cual se advierte una amplia dispersión de puntos, reflejo de la existencia de comunas con índices de pobreza y RA heterogéneos. Ocho años después (1998), la dispersión de puntos disminuye, observándose una mayor homogeneidad en el RA, sin embargo, las comunas mantienen una alta heterogeneidad en el índice de pobreza. Por último, en el año 2006 la dispersión de puntos es menor que en las dos figuras anteriores, observándose una mayor homogeneidad en el RA e índice de pobreza de las comunas. En síntesis, la secuencia de gráficos ilustra una reducción progresiva tanto del índice de pobreza como del RA comunal conforme transcurre el tiempo (i.e. mayor homogeneidad entre las comunas), reflejo de una disminución en la brecha de desigualdad.

\section{Discusión}

El presente estudio demuestra una reducción de la desigualdad en la TMI en una muestra de comunas chilenas durante el pe- ríodo 1990-2006, hecho que ocurrió en forma concomitante a una reducción de los niveles de pobreza. Durante las últimas décadas Chile ha experimentado profundas mejorías en la provisión de cuidados de salud, reflejadas en indicadores que sitúan al país en posiciones de vanguardia en el contexto latinoamericano. Sin embargo, aún persisten importantes desigualdades intercomunales, las cuales permanecen ocultas al analizar indicadores de tipo global. Una situación extrema la constituye la comparación entre las comunas incluidas en el presente estudio con el mayor y menor NSE en 2006 (Las Condes y San Bernardo, respectivamente). En 1990, la TMI de la comuna de Las Condes fue 8,5 por $1000 \mathrm{NV}$, siendo inferior a la registrada 16 años después en la comuna de San Bernardo (9,9 por 1000 NV). Transversalmente, en 2006 la TMI de la comuna de Las Condes fue similar a la registrada en Alemania (4,3 por $1000 \mathrm{NV}$, lugar $14^{\circ}$ en el mundo), mientras que la TMI de San Bernardo fue similar a la registrada en Costa Rica (9,9 por 1000 $\mathrm{NV}$, lugar $55^{\circ}$ en el mundo) $)^{9}$. Paradojal resulta el hecho de que ambas comunas se encuentran en la misma ciudad, separadas por escasos 30 kilómetros de distancia (figura 1).

La reducción de la desigualdad en la TMI en Chile está asociada directamente a una reducción de la brecha de pobreza. Al comparar el índice de pobreza de Chile y de la comuna de Las Condes, en términos absolutos la diferencia entre ambos fue de 34 puntos porcentuales en 1990, reduciéndose a 11 puntos porcentuales en 2006. Sin embargo, al utilizar un indicador de desigualdad relativo (i.e. RT) es posible verificar comportamientos en sentido opuesto. Ejemplo de ello lo constituye nuevamente la comparación entre las comunas de Las Condes y San Bernardo. Entre 1990 y 2006, el RA de la comuna de San Bernardo se redujo de 8,7 a 5,5 por $1000 \mathrm{NV}$, sin embargo, en el mismo período la RT entre ambas comunas aumentó de 2,03 a 2,27. Estos datos, aparentemente contradictorios, confirman la importancia de evaluar la desigualdad utilizando más de un indicador, en lo posible algunos de tipo absoluto y otros de tipo relativo. En este caso en particular, pese a haber aumentado la desigualdad entre ambas comunas en 
términos relativos, esta situación ocurrió en un contexto de reducción de la misma en términos absolutos, encontrándose ambas comunas (por separado) en una mejor condición que aquella registrada 16 años antes. La asociación directa entre el nivel de pobreza y la magnitud de la desigualdad en la TMI se reflejó también al comparar temporalmente el RA de diferentes comunas de la Región Metropolitana. A medida que transcurrió el tiempo, las comunas tornáronse más homogéneas en términos de su RA e índice de pobreza, confluyendo hacia un punto común de la gráfica (figura 2).

El presente estudio no es ajeno a la existencia de diversas limitaciones. Por una parte, los criterios de inclusión empleados (i.e. población mayor a 175 mil h en 2006) hicieron posible trabajar con tasas estables, decisión fundamentada por el hecho de que en 2003 en un $25 \%$ de las comunas de Chile no hubo muertes de niños menores de 1 año ${ }^{3}$. Por su parte, el hecho de ser comunas pertenecientes a la Región Metropolitana permitió darles cierta homogeneidad respecto a otros factores diferentes a su nivel de pobreza (e.g. ruralidad, cercanía a centros de alta complejidad). No obstante ello, estos criterios restan validez externa al estudio toda vez que sus resultados quieran ser extrapolados al resto de las comunas del país. Al respecto, es razonable pensar que la desigualdad en relación al estándar de referencia (i.e. comuna de Las Condes) pudiese ser mucho mayor en comunas pequeñas, rurales y de limitado acceso a centros de mayor complejidad. Este planteamiento es avalado por el análisis de la TMI de 336 comunas chilenas realizado en 2003 por Kaempffer, en el cual se reporta que 36 comunas duplicaron la TMI nacional y 6 comunas la cuadriplicaron ${ }^{3}$. Al respecto, la conducción de estudios similares al presente que incluyan comunas a lo largo de todo Chile posiblemente verificará la existencia de desigualdades de mayor magnitud.
Finalmente, esperamos que el presente estudio sea una contribución para el diseño de estrategias que permitan reducir aún más la TMI en nuestro país, así como las inequidades existentes entre comunas. La identificación de aquellas comunas con las mayores TMI (y, por consiguiente, con los mayores niveles de inequidad) constituye una herramienta de altísimo valor para focalizar intervenciones en los sectores más vulnerables de la población. Sin lugar a dudas, este desafío es uno de las más importantes que enfrenta nuestro país, ya próximo a la celebración de su Bicentenario.

\section{Referencias}

1.- Castillo-Salgado C: Medición de las desigualdades en salud. Rev Panam Salud Pública 2002; 12: 371-2.

2.- Starfield B: Equity and Health: a perspective of nonrandom distribution of Health in the population. Rev Panam Salud Pública 2002; 12: 384-7.

3.- Kaempffer AM, Medina E: Mortalidad infantil reciente en Chile: éxitos y desafíos. Rev Chil Pediatr 2006; 77 : 492-500.

4.- Donoso E: Desigualdad en mortalidad infantil entre las comunas de la provincia de Santiago. Rev Med Chile 2004; 132: 461-6.

5.- Ministerio de Salud, Chile: Objetivos Sanitarios para la Década 2000-2010. Disponible en: http://epi.minsal.cl/ Consultado el 26/11/09.

6.- Schneider MC, Castillo-Salgado C, Bacallao J, et al: Métodos de medición de las desigualdades de salud. Rev Panam Salud Pública 2002; 12: 398-414.

7.- Instituto Nacional de Estadística: Anuario de Estadísticas Vitales. Chile. 1990-2006.

8.- Ministerio de Planificación y Cooperación: Encuesta de Caracterización Socioeconómica Nacional (Casen), Chile 1990-2006. Disponible en: http://www.mideplan. cl/ Consultado el 26/11/09.

9.- United Nations, Department of Economic and Social Affairs, Population Division: World Population Prospects: The 2006 Revision, Highlights, Working Paper No. ESA/P/WP.202. 2007. 